\title{
Rising costs of health care and pathology: cause or solution?
}

\author{
J. Han van Krieken
}

Published online: 25 November 2011

(C) Springer-Verlag 2011

All over the world, there is worry about the rising costs of health care, especially in aging countries. We see much more demand from the population, much more expectations, and indeed the success of medicine in general supports that. We are able to cure diseases that were previously deadly, we can relieve symptoms even when there is no cure, we can perform surgery in situations that were previously not possible, etc. Of course this drives up the costs, and it is well accepted that the increase in costs for health care is due to its success.

This is especially the case for cancer. Survival is improving, more effective drugs have become available, and more patients present with cancer; it is essentially a disease of the elderly.

The costs of pathology are rising too: we perform more and more tests, where previously a diagnosis was based on morphology alone, and these tests, especially molecular tests, are expensive. Or are they? Pathology takes up 2-3\% of a budget of a hospital, a very modest sum. This has good and bad sides. Pathology is not expensive and brings a lot of value for money. But it is also not expensive enough to be the subject of attention for financial bodies in hospitals: there are other areas where money is spent in much larger quantities. Investments for pathology are nowhere near to what it costs to buy MRI scanners or radiotherapy machinery. To be taken seriously by management, we need to stand up and explain that actually we can be influential for cost savings.

J. H. van Krieken ( $\square)$

Department of Pathology,

Radboud University Nijmegen Medical Center, P.O. Box 9101, 6500 HB, Nijmegen, The Netherlands e-mail: j.vankrieken@pathol.umcn.nl
For a patient with cancer, a correct diagnosis as soon as possible is not only the best way to improve chances for optimal survival, it also lowers costs enormously. In case a wrong diagnosis is made, it is likely that a wrong treatment is given: spending which is not beneficial to the patient and unnecessary. In hematopathology this is actually not so rare as several recent articles have indicated a wrong diagnosis for patients with lymphoma occurs in $10-20 \%$ of cases. Readers of the Journal of Hematopathology know this all well, but not so much the financial managers of hospitals. Based on literature data, it is clear that review of lymphoma diagnosis by specialized pathologists results in more reliable diagnosis and thus better treatment. This benefits not only the patient (the most important of course) but also reduces costs to a large extent. And this review sometimes includes relatively costly methods. That is costly compared to other pathology tests, but amounting to almost nothing when compared with the modern personalized treatments.

Where does this all bring us? We need to convince hospital management, health insurance companies, and other stakeholders that it is wise to invest in pathology, especially hematopathology, because indeed a good diagnosis at the starts is saving a lot of money. We do not have to convince patients, they know very well that a correct diagnosis is a crucial moment at the beginning of their road towards cure for a cancer patient.

We, hematopathologists, need to be optimally informed and need to master our techniques, and I hope that this journal is helpful in that respect. We need to convince all others involved that indeed good pathology is one of the solutions for preventing costs for health care to run out of control and thus worth investing in. 